Article

\title{
Revealing the Bonding Nature in an ALnZnTe 3 -Type Alkaline-Metal (A) Lanthanide (Ln) Zinc Telluride by Means of Experimental and Quantum-Chemical Techniques
}

\author{
Katharina Eickmeier and Simon Steinberg * \\ Institute of Inorganic Chemistry, RWTH Aachen University, Landoltweg 1, D-52074 Aachen, Germany; \\ katharina.eickmeier@rwth-aachen.de \\ * Correspondence: simon.steinberg@ac.rwth-aachen.de; Tel.: +49-241-80-92075
}

Received: 17 September 2020; Accepted: 8 October 2020; Published: 10 October 2020

\begin{abstract}
Tellurides have attracted an enormous interest in the quest for materials addressing future challenges, because many of them are at the cutting edge of basic research and technologies due to their remarkable chemical and physical properties. The key to the tailored design of tellurides and their properties is a thorough understanding of their electronic structures including the bonding nature. While a unique type of bonding has been recently identified for post-transition-metal tellurides, the electronic structures of tellurides containing early and late-transition-metals have been typically understood by applying the Zintl-Klemm concept; yet, does the aforementioned formalism actually help us in understanding the electronic structures and bonding nature in such tellurides? To answer this question, we prototypically examined the electronic structure for an alkaline metal lanthanide zinc telluride, i.e., $\mathrm{RbDyZnTe}_{3}$, by means of first-principles-based techniques. In this context, the crystal structures of $\mathrm{RbLnZnTe}_{3}(\mathrm{Ln}=\mathrm{Gd}, \mathrm{Tb}$, Dy), which were obtained from high-temperature solid-state syntheses, were also determined for the first time by employing $\mathrm{X}$-ray diffraction techniques.
\end{abstract}

Keywords: polar intermetallics; tellurides; chemical bonding analysis

\section{Introduction}

Because of their remarkable chemical and physical properties, chalcogenides, which comprise at least one chalcogen atom in a reduced oxidation state relative to its elemental form, have gained great interest in the quest for materials that are exploited in technologies to address future challenges [1]. Among the family of chalcogenides, tellurides are of particular interest, because several tellurides are at the forefront of technologies and basic research, including explorative efforts in charge-density-waves [2-4], phase-change data storage devices [5,6], thermoelectrics $[7,8]$, and topological insulators [9], to name a few. In light of such a relevance of tellurides for basic research, it is also mandatory to determine and understand their electronic structures, because the knowledge of the electronic structure for a given material provides valuable information regarding its chemical and physical properties [10]. Hence, understanding the electronic structures of solid-state materials including tellurides ultimately also allows us to tailor their properties, as demonstrated by different high-throughput computational material design approaches [11]. Accordingly, how can the electronic structures of tellurides be described?

More recent explorative efforts on tellurides, which contain (post-)transition elements and were assigned to the family of "Incipient Metals", revealed an exclusive bonding mechanism being a combination of electron localization and delocalization [12,13]. In the cases of tellurides comprising group-I/II-elements and transition metals, the electronic structures have typically been interpreted by 
applying the Zintl-Klemm concept [14,15]. This idea was originally [16] employed to describe the (formal) valence-electron transfers from the more electropositive to the more electronegative main group elements in intermetallics. From the applications [17,18] of the Zintl-Klemm concept, it was also concluded that the more electronegative elements are arranged in clusters and fragments that are typically encountered for elements being isoelectronic to these so-called Zintl anions. In connection with applications of the Zintl-Klemm idea, it was also inferred that rare-earth-metal-tellurium interactions should be described as ionic [19-21]; however, more recent research [22-24] on lanthanide-tellurium bonding showed that lanthanides rather act as $d$-elements, like the remaining transition metals, and not as electron-donors like, for instance, alkali metals.

An additional examination [22,24-26] of the electronic structures for such binary, ternary, and quaternary tellurides, which contain copper or silver, revealed that the late-transition-metaltellurium interactions change from bonding to antibonding states below the respective valence band maxima. This outcome was in good agreement with previous research proposing a covalent bonding nature [27] and occupied antibonding states in the framework of closed-shell interactions [28,29] for such late-transition-metal-tellurium contacts. At this point, one may wonder how such latetransition-metal-tellurium interactions may emerge for late-transition metals being electron-richer than copper and silver. To answer this question, we examined the electronic structure for a previously unknown quaternary alkali metal lanthanide zinc telluride, i.e., $\mathrm{RbDyZnTe}_{3}$, that was obtained from high-temperature solid-state reactions. In doing so, we were able to quantitatively compare the zinc-tellurium interactions to the lanthanide-tellurium and alkali-metal-tellurium interactions, which were expected to be mixed-metal-like and ionic, respectively (please note that the term "mixed-metal-like" refers to late-transition-post-transition-metal interactions, whose bonding nature is similar to that of transition-metal-tellurium interactions). Herein, we present the results of these explorations and provide the very first structure report for the isostructural $\mathrm{RbLnZnTe}_{3}(\mathrm{Ln}=\mathrm{Gd}, \mathrm{Tb}, \mathrm{Dy})$.

\section{Materials and Methods}

\subsection{Syntheses}

All sample preparations were conducted under a dry argon atmosphere within a glove box (MBraun ${ }^{\circledR}$, Garching, Germany; $\mathrm{O}_{2}<0.1$ ppm by volume; $\mathrm{H}_{2} \mathrm{O}<0.1 \mathrm{ppm}$ by volume) to prevent any oxidation of the used chemicals as well as the obtained products being sensitive against air and moisture. The starting materials were powders of $\mathrm{RbCl}$ (Sigma Aldrich ${ }^{\circledR}$, St. Louis, MO, USA, 99.8\%), the respective lanthanides (smart-elements ${ }^{\circledR}$, Vienna, Austria, Dy: 99.99\%, Tb: 99.95\%; abcr ${ }^{\circledR}$, Karlsruhe, Germany, Gd: 99.9\%), zinc (laborhandel.de, Hüfingen, Germany, 99.995\%), and tellurium (Merck ${ }^{\circledR}$, Darmstadt, Germany, $>99 \%$ ), which were weighted in the initial ratios of $0.84 \mathrm{mmol} \mathrm{RbCl}$, $0.35 \mathrm{mmol} \mathrm{Zn}, 0.70 \mathrm{mmol} \mathrm{Ln}$, and $1.40 \mathrm{mmol} \mathrm{Te}$. Powders of the lanthanides were obtained from filing larger ingots, whose surfaces were polished prior to every use, while all traces of water were removed from rubidium chloride prior to the transfer into the glove box. The latter was employed as reactive flux-an approach typically [30] employed to synthesize such quaternary tellurides. The reaction mixtures were first finely ground and then loaded into one-side closed silica tubes, which were flame-sealed under a dynamic vacuum of at least $2 \times 10^{-3} \mathrm{mbar}$. The reaction mixtures were heated utilizing computer-controlled tube furnaces and the following temperature program: heat to $850^{\circ} \mathrm{C}$ within $30 \mathrm{~h}$, keep that temperature for $96 \mathrm{~h}$, slowly cool down to $300^{\circ} \mathrm{C}$ with a rate of $4{ }^{\circ} \mathrm{C} / \mathrm{h}$, and, finally, equilibrate to room temperature within $3 \mathrm{~h}$. The obtained samples were grey or red-grey powders with metallic luster and contained small crystals, which were selected for further X-ray diffraction experiments (see Section 2.2). Phase analyses based on powder X-ray diffraction patterns, which were collected for the diverse, obtained samples, revealed that the quaternary tellurides were obtained in measurable yields (see Figure 1). 


\subsection{X-ray Diffraction Studies and Crystal Structure Determinations}

To determine the yields of the quaternary tellurides and feasible by-products, phase analyses were accomplished based on the powder X-ray diffraction patterns collected for the diverse samples. To do so, the obtained samples were first ground and then loaded into capillaries, which were subsequently sealed and transferred to the used powder X-ray diffractometers. Alternatively, the ground samples were fixed between Mylar sheets with the aid of grease and then sealed in sample holders, which were subsequently transferred to the employed powder X-ray diffractometers. The sets of powder X-ray diffraction data (Table 1) were collected at room temperature with the aid of STOE ${ }^{\circledR}$ StadiP diffractometers (Stoe ${ }^{\circledR}$ \& Cie, Darmstadt, Germany; $\mathrm{Cu} \mathrm{K} \alpha_{1}$ radiation; $\lambda=1.54059 \AA$ ) ), which were equipped with area and DECTRIS ${ }^{\circledR}$ MYTHEN detectors, respectively. The WinXPow software package [31] was utilized to control the respective measurements and to further process the obtained raw data, while the phase analyses were carried out by comparing the experimentally determined powder X-ray diffraction patterns of the samples to those simulated for the quaternary tellurides as well as all possible side-products. The comparisons of the collected powder X-ray diffraction patterns to those which were simulated for the quaternary tellurides and the side-products were conducted with the programs WinXPow and Match! [32], respectively.

Table 1. Details of the crystal structure investigations and refinements for $\operatorname{RbLnZnTe}_{3}(\mathrm{Ln}=\mathrm{Gd}, \mathrm{Tb}, \mathrm{Dy})$.

\begin{tabular}{|c|c|c|c|}
\hline Formula & $\mathrm{RbGdZnTe}_{3}$ & $\mathrm{RbTbZnTe}_{3}$ & $\mathrm{RbDyZnTe}_{3}$ \\
\hline form wt. & 690.89 & 692.56 & 696.14 \\
\hline space group & & $\mathrm{Cmcm}$ & \\
\hline$a(\AA)$ & $4.419(1)$ & $4.395(1)$ & $4.410(2)$ \\
\hline$b(\AA)$ & $16.072(5)$ & $16.058(5)$ & $16.144(6)$ \\
\hline$c(\AA)$ & $11.716(4)$ & $11.651(4)$ & $11.671(4)$ \\
\hline volume $\left(\AA^{3}\right)$ & $832.0(4)$ & $822.3(4)$ & $831.0(5)$ \\
\hline Z & 4 & 4 & 4 \\
\hline density (calc.), $\mathrm{g} / \mathrm{cm}^{3}$ & 5.515 & 5.594 & 5.564 \\
\hline$\mu\left(\mathrm{mm}^{-1}\right)$ & 26.797 & 27.648 & 27.842 \\
\hline$F(000)$ & 1148 & 1152 & 1156 \\
\hline \multirow[t]{2}{*}{$\theta$ range $\left(^{\circ}\right)$} & $2.53-25.64$ & $2.54-25.65$ & $2.52-25.62$ \\
\hline & $-5 \leq h \leq 5$ & $-5 \leq h \leq 5$ & $-5 \leq h \leq 5$ \\
\hline \multirow[t]{2}{*}{ index range } & $-15 \leq k \leq 19$ & $-19 \leq k \leq 16$ & $-19 \leq k \leq 19$ \\
\hline & $-14 \leq l \leq 14$ & $-14 \leq l \leq 13$ & $-12 \leq l \leq 14$ \\
\hline reflections collected & 2031 & 2385 & 2367 \\
\hline independent reflections & 473 & 468 & 473 \\
\hline refinement method & & Full matrix least squares on $F^{2}$ & \\
\hline data/restraints/parameters & $473 / 0 / 23$ & $468 / 0 / 23$ & $473 / 0 / 23$ \\
\hline goodness-of-fit on $F^{2}$ & 1.176 & 1.190 & 1.258 \\
\hline final $R$ indices $[I>2 \sigma(I)]$ & $R_{1}=0.019 ; w R_{2}=0.043$ & $R_{1}=0.018 ; w R_{2}=0.036$ & $R_{1}=0.032 ; w R_{2}=0.079$ \\
\hline$R$ indices (all data) & $R_{1}=0.020 ; w R_{2}=0.044$ & $R_{1}=0.018 ; w R_{2}=0.036$ & $R_{1}=0.032 ; w R_{2}=0.079$ \\
\hline$R_{\text {int }}$ & 0.024 & 0.020 & 0.040 \\
\hline $\begin{array}{c}\text { largest diff. peak and } \\
\text { hole, } \mathrm{e}^{-} / \AA^{3}\end{array}$ & 1.077 and -0.900 & 0.667 and -0.922 & 2.051 and -2.067 \\
\hline
\end{tabular}



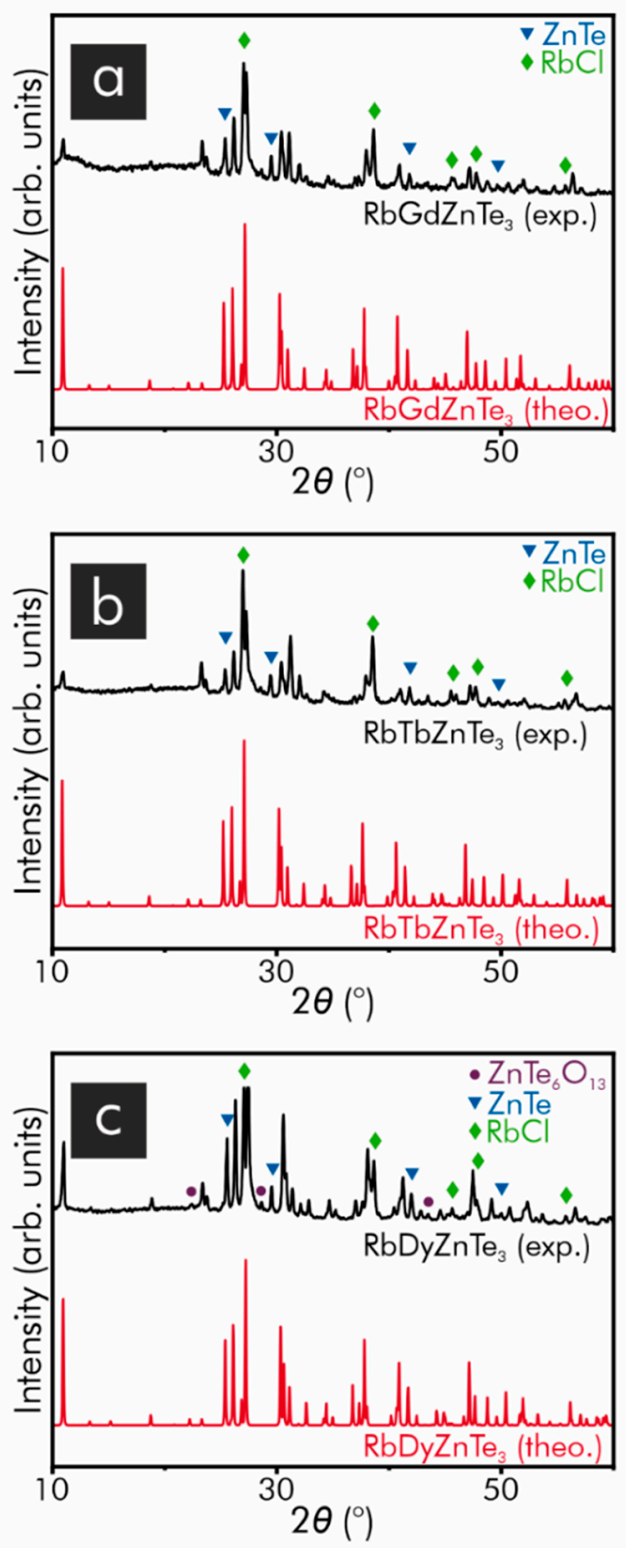

Figure 1. Measured and simulated powder X-ray diffraction patterns of (a) $\operatorname{RbGdZnTe}_{3},(\mathbf{b}) \mathrm{RbTbZnTe}_{3}$, and (c) $\mathrm{RbDyZnTe}_{3}$ : reflections arising from the side-products $\mathrm{RbCl}$ [33], $\mathrm{ZnTe}$ [34], and $\mathrm{ZnTe}_{6} \mathrm{O}_{13}$ [35] are marked by the diverse symbols. A closer inspection of the measured powder X-ray diffraction patterns also points to the presence of additional reflections $\left(\sim 25^{\circ}\right)$, which correspond to unknown phases.

For the determinations of the crystal structures of the quaternary tellurides, sets of single-crystal X-ray intensity data were collected at room temperature by utilizing a Bruker ${ }^{\circledR}$ APEX CCD diffractometer (Bruker Inc. ${ }^{\circledR}$, Madison, WI, USA; Mo K $\alpha$ radiation; $\lambda=0.71073 \AA$ ). Prior to the collection of the single-crystal X-ray diffraction datasets, samples were first selected from the bulk materials and then fixed in capillaries, which were sealed and transferred to the aforementioned diffractometer for initial inspections of the quality of the selected samples and the collection of the single-crystal X-ray intensity datasets. The integrations of the sets of raw data and the multi-scan absorption corrections were conducted using the programs [36] SAINT+ and SADABS, respectively, while identifying the reflection conditions for the single-crystal $\mathrm{X}$-ray intensity datasets was accomplished by means of the XPREP code [37], which suggested the space group Cmcm (no. 63) for the tellurides. The aforementioned space group was used for the initial structure solutions based on applying direct methods (SHELXS-97 [38]), while the structures were refined in full matrix least-squares of 
$F^{2}$ (SHELXL-97 [38,39]), including anisotropic displacement parameters (Table 2). CCDC 2031653, 2031660, and 2031661 contain the crystallographic data for this paper. These data can be obtained free of charge via http://www.ccdc.cam.ac.uk/conts/retrieving.html or from the CCDC, 12 Union Road, Cambridge CB21EZ; Fax: +44-1223-336033; E-Mail: deposit.ccdc.cam.ac.uk.

Table 2. Atomic positions and equivalent displacement parameters for $\operatorname{RbLnZnTe}_{3}(\mathrm{Ln}=\mathrm{Gd}, \mathrm{Tb}, \mathrm{Dy})$.

\begin{tabular}{cccccc}
\hline Atom & Position & $x$ & $y$ & $z$ & $U_{\text {eq }}, \AA^{2}$ \\
\hline & & \multicolumn{5}{c}{$\operatorname{RbGdZnTe}_{3}$} \\
$\mathrm{Gd} 1$ & $4 a$ & 0 & 0 & 0 & $0.0147(2)$ \\
$\mathrm{Te} 1$ & $8 f$ & 0 & $0.37160(3)$ & $0.06048(4)$ & $0.0156(2)$ \\
$\mathrm{Te} 2$ & $4 c$ & 0 & $0.06418(4)$ & $\frac{1}{4}$ & $0.0135(2)$ \\
$\mathrm{Rb} 1$ & $4 c$ & 0 & $0.74613(7)$ & $\frac{1}{4}$ & $0.0304(3)$ \\
$\mathrm{Zn} 1$ & $4 c$ & 0 & $0.46104(8)$ & $\frac{1}{4}$ & $0.0184(3)$ \\
& & & $\mathrm{RbTbZnTe} 3$ & & \\
$\mathrm{~Tb} 1$ & $4 a$ & 0 & 0 & 0 & $0.0149(1)$ \\
$\mathrm{Te} 1$ & $8 f$ & 0 & $0.37212(2)$ & $0.05945(3)$ & $0.0157(1)$ \\
$\mathrm{Te} 2$ & $4 c$ & 0 & $0.06422(3)$ & $\frac{1}{4}$ & $0.0138(1)$ \\
$\mathrm{Rb} 1$ & $4 c$ & 0 & $0.74653(5)$ & $\frac{1}{4}$ & $0.0301(2)$ \\
$\mathrm{Zn} 1$ & $4 c$ & 0 & $0.46119(6)$ & $\frac{1}{4}$ & $0.0186(2)$ \\
& & & $\mathrm{RbDyZnTe}$ & & \\
$\mathrm{Dy} 1$ & $4 a$ & 0 & 0 & 0 & $0.0123(3)$ \\
$\mathrm{Te} 1$ & $8 f$ & 0 & $0.37263(4)$ & $0.05871(6)$ & $0.0131(3)$ \\
$\mathrm{Te} 2$ & $4 c$ & 0 & $0.06422(6)$ & $\frac{1}{4}$ & $0.0114(3)$ \\
$\mathrm{Rb} 1$ & $4 c$ & 0 & $0.74683(10)$ & $\frac{1}{4}$ & $0.0279(4)$ \\
$\mathrm{Zn} 1$ & $4 c$ & 0 & $0.46143(11)$ & $\frac{1}{4}$ & $0.0161(4)$ \\
\hline
\end{tabular}

\subsection{Computational Details}

To provide an insight into the electronic structure and bonding nature for the ALnZnTe 3 -type tellurides, we prototypically examined the electronic structure of $\mathrm{RbDyZnTe}_{3}$. Prior to the bonding analysis, full structural optimizations, which included lattice parameters and atomic positions, were accomplished using the projector augmented wave method [40] that was also employed for the electronic band structure computations and implemented in the Vienna ab-initio simulation package [41-45] (VASP). In all computations, correlation and exchange were described by the generalized gradient approximation of Perdew, Burke, and Ernzerhof (GGA-PBE) [46], and the energy cut-off of the plane-wave basis sets was $500 \mathrm{eV}$. A $24 \times 6 \times 8 \mathrm{k}$-points set was used to sample the first Brillouin zone, and all computations were considered to be converged, as the energy difference between two iterative steps fell below $10^{-8} \mathrm{eV} / \mathrm{cell}$ (and $10^{-6} \mathrm{eV} / \mathrm{cell}$ ) for the electronic (and ionic) relaxations. The dysprosium $4 f$ states were treated as core-like in all computations-an approach that can be adopted because the valence electrons residing in the $4 f$ states are typically localized and, hence, barely contribute to overall bonding in a given solid-state material [47-49].

A bonding analysis was completed based on the projected crystal orbital Hamilton population approach [50] (pCOHP) that is a variant of the COHP method [51,52]. Within the latter approach, the off-site entries of the densities-of-states (DOS) matrix of a given material are weighted with the corresponding Hamilton matrix elements to unfold bonding, non-bonding, and antibonding states. Because the crystal orbitals employed in the COHP approach require the uses of basis sets, in which the electronic structures correspond to a local nature instead of a delocalized one as within plane-wave representations, the outcome of the plane-wave-based computations had to be transformed in all electron contracted Slater-type orbitals. The aforementioned transformations were accomplished by means of the Local Orbital Basis Suite Towards Electronic-Structure Reconstruction (LOBSTER) program $[50,51,53,54]$, in which transfer matrices are applied to the results of plane-wave-based computations in order to construct the crystal wave functions. In doing so, we also determined the gross populations for all atoms and subtracted them from the respective valence electron counts to 
obtain the Mulliken and Löwdin charges [55]. Representations of the DOS and the pCOHPs were generated with the aid of the wxDragon [56] code, shown in Figure 2.

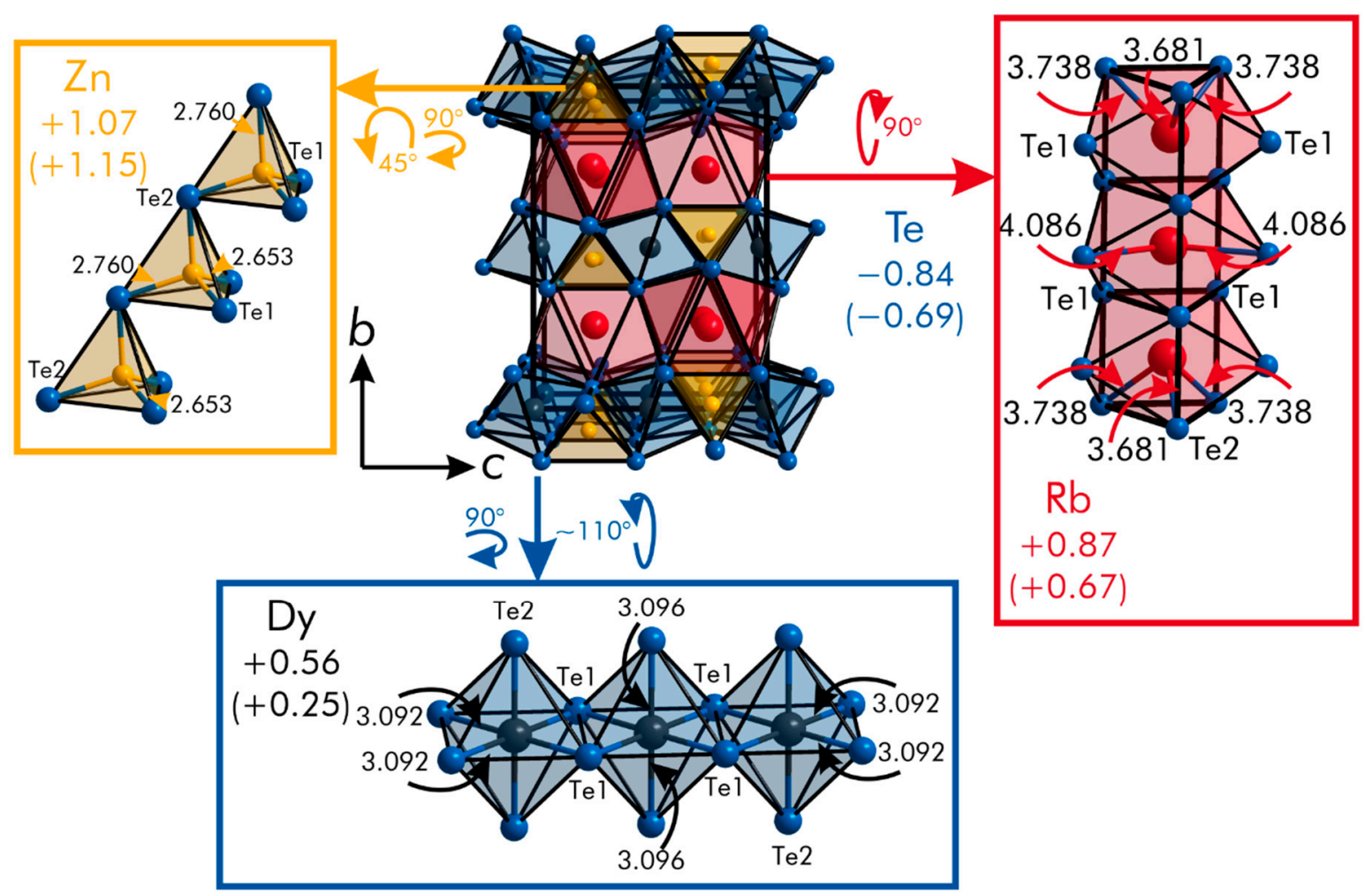

Figure 2. Representation of the crystal structure of the ALnZnTe 3 -type tellurides: the different types of tellurium polyhedra enclosing the alkaline metals, lanthanides, and zinc atoms, respectively, are shown in the insets, while the Mulliken and Löwdin (in parentheses) charges computed for $\mathrm{RbDyZnTe}_{3}$ have been included. The Mulliken and Löwdin charges of tellurium correspond to the averaged charges of both crystallographically independent tellurium sites, for which the charges solely differ by $0.05 \mathrm{e}$.

\section{Results and Discussion}

$\mathrm{RbLnZnTe}_{3}(\mathrm{Ln}=\mathrm{Gd}, \mathrm{Tb}$, Dy) were obtained from high-temperature reactions of rubidium chloride, zinc, tellurium, and the respective lanthanides (see Section 2.1) and crystallized with the $\mathrm{KCuZrS}_{3}$-type [57] of structure (Pearson Symbol oS24; $\mathrm{Z}=4$ ), which has been frequently [58] adopted by quaternary alkaline-metal early-transition-metal late-transition-metal chalcogenides. A comparison of the volumes of the unit cells of $\operatorname{RbLnZnTe}_{3}(\mathrm{Ln}=\mathrm{Gd}, \mathrm{Tb}$, Dy; Table 1) to those of the isostructural cesium-containing [59] tellurides $\left(V_{\mathrm{CsGdZnTe}_{3}}=863.55 \AA^{3} ; V_{\mathrm{CsTbZnTe}_{3}}=857.10 \AA^{3}\right.$; $V_{\mathrm{CsDyZnTe}_{3}}=851.11 \AA^{3}$ ) shows that the unit cell volumes of the latter are larger than those of the former. This is because of the decreased covalent radius [60] from cesium $(2.44 \AA)$ to rubidium $(2.20 \AA)$; yet, the unit cell volumes within the series $\operatorname{RbLnZnTe}_{3}(\mathrm{Ln}=\mathrm{Gd}, \mathrm{Tb}$, Dy) solely exhibit a very small variation-a circumstance that has also been encountered for different polar intermetallics containing heavier lanthanides [24,61-63]. In the following, the crystal structure of this type of quaternary telluride will be prototypically depicted for RbDyZnTes .

An inspection of the $\mathrm{RbDyZnTe}_{3}$ crystal structure reveals that each unit cell comprises one rubidium position (Wyckoff site 4c), one zinc position (Wyckoff site 4c), and one dysprosium position (Wyckoff site $4 a$ ), which are enclosed by diverse types of tellurium polyhedra. The zinc atoms are encompassed by the tellurium atoms in a tetrahedral fashion within $\left[\mathrm{Zn} @ \mathrm{Te}_{4}\right]$ units, while the dysprosium atoms are located in the centers of tellurium octahedra as represented by the formula [Dy@ $\mathrm{Te}_{6}$ ] (note that the indices of the tellurium atoms refer to the respective coordination numbers in the diverse tellurium units). Each [Dy@ $\left.\mathrm{Te}_{6}\right]$ unit shares two common edges with the two nearest 
neighboring [Dy@ $\left.\mathrm{Te}_{6}\right]$ units within one linear ${ }_{\infty}^{1}\left[\mathrm{Dy} @ \mathrm{Te}_{6}\right]$ chain, which is connected via common tellurium vertices to neighboring ${ }_{\infty}^{1}\left[\mathrm{Dy} @ \mathrm{Te}_{6}\right]$ chains. These linear chains of tellurium octahedra enclosing the dysprosium atoms are tilted relative to each other such that the sheets of the ${ }_{\infty}^{1}\left[\mathrm{Dy} @ \mathrm{Te}_{6}\right]$ chains propagating parallel to the $a$ and $c$ axes are puckered.

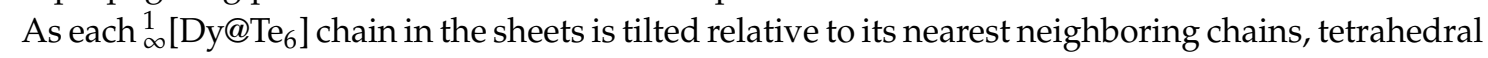
voids open up between the chains of the octahedral tellurium units enclosing the dysprosium atoms. These tetrahedral voids are occupied by the zinc atoms, while each tetrahedral [ $\left.\mathrm{Zn} @ \mathrm{Te}_{4}\right]$ unit is connected via two common tellurium vertices to two neighboring $\left[\mathrm{Zn} @ \mathrm{Te}_{4}\right]$ units within a linear ${ }_{\infty}^{1}\left[\mathrm{Zn} @ \mathrm{Te}_{4}\right]$ chain. The puckered sheets of the ${ }_{\infty}^{1}\left[\mathrm{Zn} @ \mathrm{Te}_{4}\right]$ and ${ }_{\infty}^{1}\left[\mathrm{Dy} @ \mathrm{Te}_{6}\right]$ chains are separated from each other by the rubidium atoms, which, in turn, are sandwiched by the aforementioned sheets along the $b$ axis. The rubidium atoms are located in the centers of bicapped trigonal tellurium prisms, which are condensed via the trigonal bases into linear ${ }_{\infty}^{1}\left[\mathrm{Rb}_{\mathrm{T}} \mathrm{Te}_{8}\right]$ chains along the $a$ axis.

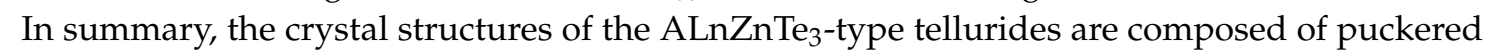
sheets of linear ${ }_{\infty}^{1}\left[\mathrm{Zn} @ \mathrm{Te}_{4}\right]$ and ${ }_{\infty}^{1}\left[\mathrm{Ln} @ \mathrm{Te}_{6}\right]$ chains and layers of ${ }_{\infty}^{1}\left[\mathrm{~A} @ \mathrm{Te}_{8}\right]$ chains that propagate parallel to the $a c$ plane and are stacked along the $b$ axis. An application of the Zintl-Klemm concept to this type of telluride points to an electron-precise valence-electron distribution according to the formula $\left(\mathrm{A}^{+}\right)\left(\mathrm{Ln}^{3+}\right)\left(\mathrm{Zn}^{2+}\right)\left(\mathrm{Te}^{2-}\right)_{3}$; however, as pointed out in the Introduction, more recent research indicated that such Zintl-Klemm treatments could be misleading for characterizing the nature of lanthanide-tellurium bonding. Furthermore, it was shown (see Introduction) that the late-transition-metal-tellurium interactions change from bonding to antibonding states below the Fermi levels for certain tellurides. Hence, how will these heteroatomic interactions emerge for a quaternary telluride containing a late-transition-metal being electron-richer relative to those inspected as part of the aforementioned examinations? To answer this question, we followed up with an examination of the electronic structure and nature of bonding for the dysprosium-containing telluride.

An examination of the densities-of-states (DOS) curves for the quaternary $\mathrm{RbDyZnTe}_{3}$ reveals that the Fermi level falls in a band gap between $0 \mathrm{eV}$ and around $1.34 \mathrm{eV}$ (Figure 3). Such a characteristic at the Fermi level of a given solid-state material is frequently [18] encountered for Zintl phases and can typically [64] be considered as an electronically favorable situation that appears also to be evident for $\mathrm{RbDyZnTe}_{3}$. An additional inspection of the DOS corresponding to the energy regions near the valence band maximum and conducting band minimum indicates that these states largely stem from the Te-5p and $\mathrm{Zn}-4 s / 3 d$ orbitals, with minor contributions from Dy- $5 d$ atomic orbitals. A comparison of the Mulliken and Löwdin charges reveals that the charges computed for rubidium are close to the charge predicted by the Zintl-Klemm treatment (see above); however, the Mulliken and Löwdin charges calculated for dysprosium, zinc, and tellurium are evidently smaller than the charges proposed by applying the Zintl-Klemm concept to $\mathrm{RbDyZnTe}_{3}$. The outcome of the Mulliken and Löwdin charge population analyses implies that nearly full valence electron transfers are evident from rubidium to the tellurium atoms such that the rubidium-tellurium separations should be regarded as ionic interactions. Because the population analyses based on the Mulliken and Löwdin charges point to a clear absence of full valence electron transfers for the dysprosium-tellurium and zinc-tellurium contacts, the bonding nature related to the aforementioned separations can barely be depicted as ionic. 


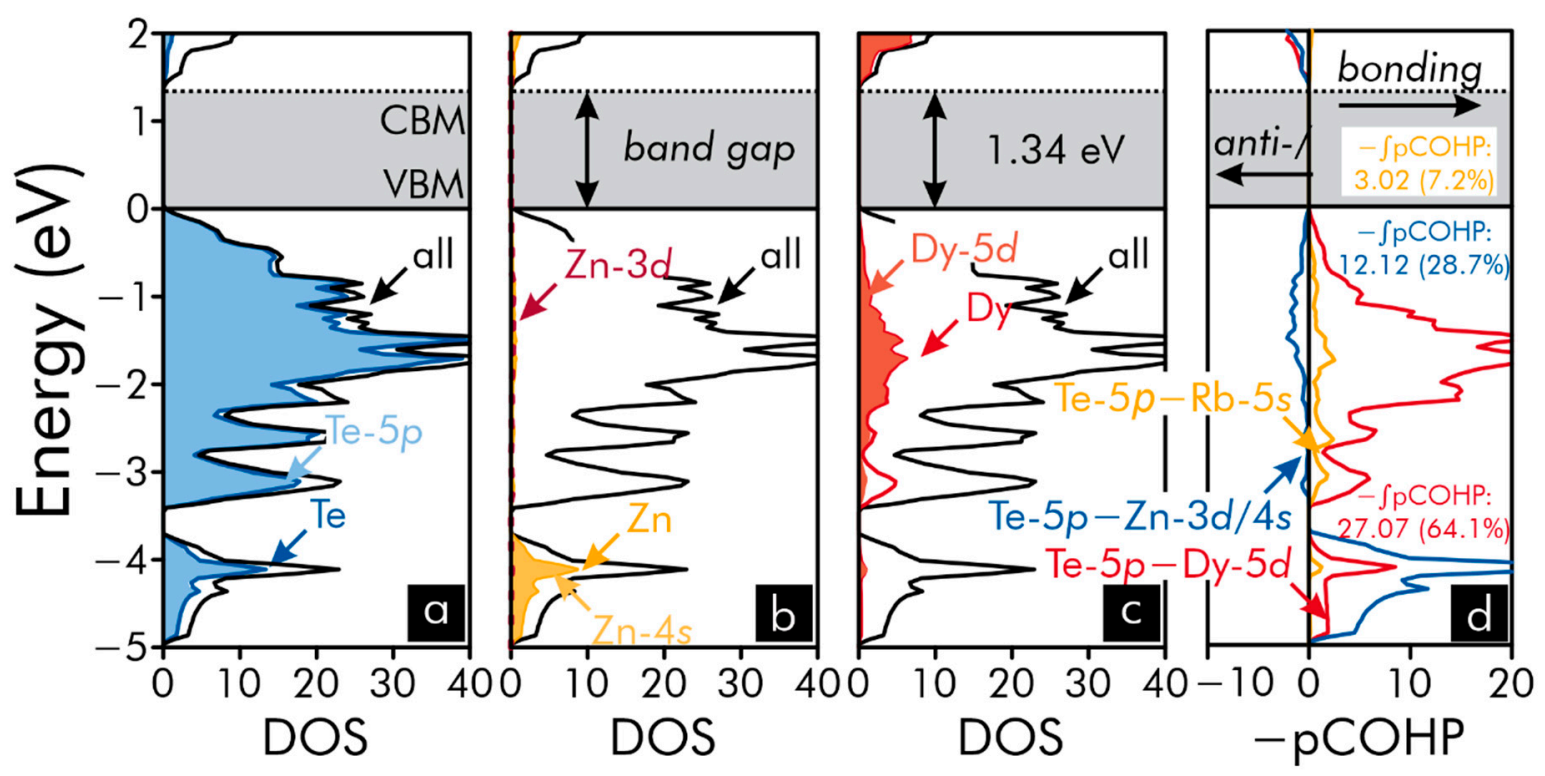

Figure 3. (a-c) Densities-of-states (DOS) curves of $\mathrm{RbDyZnTe}_{3}$ : the valence band maximum (VBM) and conducting band minimum (CBM) are represented by the lower and upper black horizontal lines, respectively, while the atom- and orbital-projected DOS are included; (d) projected crystal orbital Hamilton populations (pCOHP) of $\mathrm{RbDyZnTe}_{3}$ : the cumulative integrated values as well as percentages of every inspected interaction to the net bonding capabilities (see main text) are included.

To understand the bonding nature of $\mathrm{RbDyZnTe}_{3}$ in more detail, we followed up with an examination of the projected crystal orbital Hamilton populations (-pCOHP) of this quaternary telluride (Figure 3). In this connection, we also determined the cumulative integrated -pCOHP $(-\mathrm{IpCOHP})$ values, which are obtained by summing up all -IpCOHP/bond values for a respective sort of interatomic contact within the unit cell of a given solid-state material, and projected these values as percentages of the net bonding capabilities. This approach was chosen because previous research [52] showed that this procedure reveals the bonding nature for a given solid-state material as well as similarities and differences between the nature of bonding for solid-state materials with dissimilar compositions. In doing so, we paid particular attention to the orbital-based contributions arising from the atomic orbitals, which provide the largest contributions to the states near the Fermi level.

A comparison of the percentage contributions of the cumulative-IpCOHP values to the net bonding capabilities reveals that the smallest percentages correspond to the Rb-5s-Te-5p interactions, while the amounts of the rubidium-tellurium contacts per unit cell (32) are higher than those of the zinc-tellurium (16) and dysprosium-tellurium (24) separations. Although the rubidium-tellurium contacts are related to the highest bond frequencies among all inspected interactions, the aforementioned $\mathrm{Rb}-5 s-\mathrm{Te}-5 p$ interactions correspond to the lowest percentage contribution to the net bonding capability because of the relatively small $-\mathrm{IpCOHP} /$ bond values $\left(<-\mathrm{IpCOHP} /\right.$ bond $>_{\mathrm{Rb}-5 s-\mathrm{Te}-5 p}=0.09446 \mathrm{eV} / \mathrm{bond}$ ). Such small $-\mathrm{IpCOHP/bond} \mathrm{values} \mathrm{mean} \mathrm{that} \mathrm{these} \mathrm{states} \mathrm{are} \mathrm{less} \mathrm{populated} \mathrm{due} \mathrm{to} \mathrm{the} \mathrm{valence}$ electron transfer from the rubidium to the tellurium atoms-an outcome that is also indicated by the Mulliken and Löwdin population analyses (see above) and underlines the ionic character of the rubidium-tellurium interactions.

Because the Dy-5d-Te-5p and Zn-3d/4s-Te-5p interactions correspond to higher -IpCOHP/bond values $\left(<-\mathrm{IpCOHP} /\right.$ bond $>_{\text {Dy-5 } 5 d-\mathrm{Te}-5 p}=1.1281 \mathrm{eV} /$ bond; $<-\mathrm{IpCOHP} /$ bond $\left.>_{\mathrm{Zn}-4 s / 3 d-\mathrm{Te}-5 p}=0.7575 \mathrm{eV} / \mathrm{bond}\right)$ than the Rb-5s-Te-5p interactions, it can be inferred that the former interactions are more bonding than the latter and, hence, represent a type of bonding being different from that of the rubidium-tellurium contacts. Because a more bonding character is typically $[64,65]$ indicative of a higher degree of electron-sharing and the Mulliken and Löwdin population analyses point to certain valence electron transfers from the dysprosium and zinc to the tellurium atoms, a polar-covalent bonding character is indicated for 
the Dy-5d-Te-5 $p$ and Zn-3d/4s-Te-5p interactions. A comparison of the percentages of the cumulative -IpCOHP to the net bonding capabilities reveals that the contributions of the Dy-5d-Te-5p interactions are higher than those of the $\mathrm{Zn}-3 d / 4 s-\mathrm{Te}-5 p$ interactions, because the latter interactions show a lower bond frequency and slightly smaller - IpCOHP/bond values than the former. Accordingly, there is a more bonding character between the Te-5p and the Dy-5d orbitals than between the Te-5p and the Zn-3d/4s orbitals (notably, the $\mathrm{Zn}-3 d$ bands also show rather small dispersions at around $-7 \mathrm{eV}$ ), as the latter interactions change from bonding to antibonding states below the valence band maximum.

As such transitions from bonding to antibonding states have also been determined by previous research $[66,67]$ on zinc-post-transition-metal interactions, it could also be concluded to depict the zinc-tellurium interactions as mixed-metal-like interactions. Furthermore, it should also be noted that our bonding analysis clearly indicates that the dysprosium-tellurium interactions can hardly be described as ionic, as dysprosium rather acts as a $d$-metal [68] —an outcome that is in full agreement with our previous research on the bonding nature of lanthanide-tellurium interactions (see Introduction). At this point, one may wonder if this sort of telluride should be rather assigned to the group of the Zintl phases or the family of polar intermetallics. While the knowledge of the valence electron concentration helps us in distinguishing between Hume-Rothery and polar intermetallic phases, it is hard to arrange a frontier between Zintl phases and polar intermetallics by means of the valence electron concentration [69-71]. Because the antibonding states in Zintl phases are usually [18] unoccupied and well separated from non-bonding and bonding states, the populations of antibonding zinc-tellurium states below the valence band maximum are in stark contrast to the aforementioned electronic structure criterion of Zintl phases. Hence, the electronic structure of $\mathrm{RbDyZnTe}_{3}$ is best described as a polyanionic network, which consists of polar-covalently bonded zinc and dysprosium centered tellurium polyhedrons and monoatomic rubidium cations enclosed by the aforementioned network.

\section{Conclusions}

In the quest for materials showing the requested task-specific properties, there is a need to understand their electronic structures, including the nature of bonding, because the interpretations of the electronic structures of solid-state materials allow us to extract invaluable information regarding their chemical and physical properties. Among solid-state materials, tellurides have attracted particular interest, as many tellurides are at the cutting edge of basic research and technologies; yet, are the Zintl-Klemm treatments, which are typically carried out to understand the valence electron distributions in tellurides, helpful to understand the electronic structures and bonding nature of tellurides containing transition-metals? Furthermore, should tellurides comprising transition-metals be viewed at as Zintl phases or polar intermetallics?

To answer these questions, we prototypically examined the electronic structure and bonding nature of $\mathrm{RbDyZnTe}_{3}$, which crystallizes with the $\mathrm{KCuZrS}_{3}$-type of structure. In doing so, we also provided the first structural reports on the previously unknown $\operatorname{RbLnZnTe}_{3}(\mathrm{Ln}=\mathrm{Gd}, \mathrm{Tb}$, Dy), whose crystal structures are composed of two different types of layers that propagate parallel to the $a c$ plane and are stacked along the $b$ axis. One of the layers comprises linear chains of bicapped trigonal tellurium prisms enclosing the rubidium atoms, while the other layer consists of linear chains of tellurium tetrahedra and octahedra encompassing the zinc and dysprosium atoms, respectively. An application of the Zintl-Klemm concept to the ALnZnTe 3 -type tellurides proposes an electron-precise valence electron distribution according to the formula $\left(\mathrm{A}^{+}\right)\left(\mathrm{Ln}^{3+}\right)\left(\mathrm{Zn}^{2+}\right)\left(\mathrm{Te}^{2-}\right)_{3}$. However, the outcome of the first-principles-based examinations of the electronic structure and bonding nature of $\mathrm{RbDyZnTe}_{3}$ clearly shows that an application of the aforementioned formalism provides a rather simplified picture of the electronic structure and could also be misleading: the nature of bonding of the heteroatomic contacts between the rubidium and the tellurium atoms should be described as ionic, while the nature of the $\mathrm{Dy}-\mathrm{Te}$ and $\mathrm{Zn}-\mathrm{Te}$ interactions should be classified as polar-covalent or mixed-metal-like. This outcome is in stark contrast to an ionic description of the lanthanide-tellurium interactions, which have been previously proposed for tellurides comprising lanthanides (see Introduction). As the 
structures of these tellurides are best described as polar-covalently bonded frameworks enclosing monoatomic (alkali metal) counterions, the $\mathrm{ALnZnTe}_{3}$-type tellurides rather show characteristics of polar intermetallics and, hence, should be assigned to the family of polar intermetallics.

Author Contributions: K.E. conducted the syntheses and the structure solutions as well as refinements, while the quantum-chemical examinations were carried out by S.S., who supervised the project and prepared the first draft of the manuscript. The manuscript was written with contributions from both authors, who approved the final version of the manuscript. All authors have read and agreed to the published version of the manuscript.

Funding: This work was supported by the Verband der Chemischen Industrie (FCI, Frankfurt a.M.) through a Liebig-stipend to S.S.

Acknowledgments: We wish to thank R. Dronskowski (https://en.wikipedia.org/wiki/Richard_Dronskowski) for the allocation of the lab space and the computer cluster of the Chemistry Department of RWTH Aachen University, Tobias Storp for the collections of the sets of PXRD and SCXRD data, and Fabian Gladisch, M.Sc., for the technical assistance regarding the absorption corrections.

Conflicts of Interest: The authors declare no conflict of interest.

\section{References}

1. Kanatzidis, M.G. Discovery-Synthesis, Design, and Prediction of Chalcogenide Phases. Inorg. Chem. 2017, 56, 3158-3173. [CrossRef] [PubMed]

2. Sheldrick, W.S. Polychalcogenide Anions: Structural Diversity and Ligand Versatility. Z. Anorg. Allg. Chem. 2012, 638, 2401-2424. [CrossRef]

3. Böttcher, P.; Doert, T. Chalcogene-Rich Chalcogenides: From the First Ideas to a Still Growing Field of Research, Phosphorus, Sulfur, and Silicon and the Related Elements. Phosphorus Sulfur Silicon Relat. Elem. 1998, 136, 255-282. [CrossRef]

4. Fries, K.S.; Steinberg, S. Fermi-Level Characteristics of Potential Chalcogenide Superconductors. Chem. Mater. 2018, 30, 2251-2261. [CrossRef]

5. Wuttig, M.; Yamada, N. Phase-change materials for rewriteable data storage. Nat. Mater. 2007, 6, 824-832. [CrossRef]

6. Wuttig, M.; Raoux, S. The Science and Technology of Phase Change Materials. Z. Anorg. Allg. Chem. 2012, 638, 2455-2465. [CrossRef]

7. Toberer, E.S.; Snyder, G.J. Complex thermoelectric materials. Nat. Mater. 2008, 7, 105-114. [CrossRef]

8. Sootsman, J.R.; Chung, D.Y.; Kanatzidis, M.G. New and Old Concepts in Thermoelectric Materials. Angew. Chem. Int. Ed. 2009, 48, 8616-8639. [CrossRef]

9. Hasan, M.Z.; Kane, C.L. Colloquium: Topological Insulators. Rev. Mod. Phys. 2010, 82, 3045-3067. [CrossRef]

10. Miller, G.J. The "Coloring Problem" in Solids: How It Affects Structure, Composition and Properties. Eur. J. Inorg. Chem. 1998, 1998, 523-536. [CrossRef]

11. Curtarolo, S.; Hart, G.L.W.; Nardelli, M.B.; Mingo, N.; Sanvito, S.; Levy, O. The high-throughput highway to computational materials design. Nat. Mater. 2013, 12, 191-201. [CrossRef] [PubMed]

12. Wuttig, M.; Deringer, V.L.; Gonze, X.; Bichara, C.; Raty, J.-Y. Incipient Metals: Functional Materials with a Unique Bonding Mechanism. Adv. Mater. 2018, 30, 1803777. [CrossRef] [PubMed]

13. Raty, J.-Y.; Schumacher, M.; Golub, P.; Deringer, V.L.; Gatti, C.; Wuttig, M. A Quantum-Mechanical Map for Bonding and Properties in Solids. Adv. Mater. 2019, 31, 1806280. [CrossRef] [PubMed]

14. Böttcher, P. Tellurium-Rich Tellurides. Angew. Chem. Int. Ed. Engl. 1988, 27, 759-772. [CrossRef]

15. Papoian, G.A.; Hoffmann, R. Hypervalent Bonding in One, Two, and Three Dimensions: Extending the Zintl-Klemm Concept to Nonclassical Electron-Rich Networks. Angew. Chem. Int. Ed. 2000, 39, 2408-2448. [CrossRef]

16. Zintl, E. Intermetallische Verbindungen. Angew. Chem. 1939, 52, 1-6. [CrossRef]

17. Schäfer, H.; Eisenmann, B.; Müller, W. Zintl Phases: Transitions between Metallic and Ionic Bonding. Angew. Chem. Int. Ed. 1973, 12, 694-712. [CrossRef]

18. Miller, G.J.; Schmidt, M.W.; Wang, F.; You, T.-S. Quantitative Advances in the Zintl-Klemm Formalism. Struct. Bond. 2011, 139, 1-55. [CrossRef] 
19. Meng, C.-Y.; Chen, H.; Wang, P.; Chen, L. Syntheses, Structures, and Magnetic and Thermoelectric Properties of Double-Tunnel Tellurides: $\mathrm{A}_{\mathrm{x}} \mathrm{RE}_{2} \mathrm{Cu}_{6-\mathrm{x}} \mathrm{Te}_{6}(\mathrm{~A}=\mathrm{K}-\mathrm{Cs} ; \mathrm{RE}=\mathrm{La}-\mathrm{Nd})$. Chem. Mater. 2011, 23, 4910-4919. [CrossRef]

20. Meng, C.-Y.; Chen, H.; Wang, P. Syntheses, Structures, and Physical Properties of $\mathrm{CsRE}_{2} \mathrm{Ag}_{3} \mathrm{Te}_{5}(\mathrm{RE}=\mathrm{Pr}, \mathrm{Nd}$, $\mathrm{Sm}, \mathrm{Gd}-\mathrm{Er}$ ) and $\mathrm{RbRE}_{2} \mathrm{Ag}_{3} \mathrm{Te}_{5}$ ( $\left.\mathrm{RE}=\mathrm{Sm}, \mathrm{Gd}-\mathrm{Dy}\right)$. Inorg. Chem. 2014, 53, 6893-6903. [CrossRef]

21. Lin, H.; Chen, H.; Shen, J.-N.; Chen, L.; Wu, L.-M. Chemical Modification and Energetically Favorable Atomic Disorder of a Layered Thermoelectric Material $\mathrm{TmCuTe}_{2}$ Leading to High Performance. Chem. Eur. J. 2014, 20, 15401-15408. [CrossRef]

22. Gladisch, F.C.; Steinberg, S. Revealing the Nature of Bonding in Rare-Earth Transition-Metal Tellurides by Means of Methodes Based on First Principles. Eur. J. Inorg. Chem. 2017, 2017, 3395-3400. [CrossRef]

23. Göbgen, K.C.; Fries, K.S.; Gladisch, F.C.; Dronskowski, R.; Steinberg, S. Revealing the Nature of Chemical Bonding in an $\mathrm{ALn}_{2} \mathrm{Ag}_{3} \mathrm{Te}_{5}$-Type Alkaline-Metal (A) Lanthanide (Ln) Silver Telluride. Inorganics 2019, 7, 70. [CrossRef]

24. Eickmeier, K.; Fries, K.S.; Gladisch, F.C.; Dronskowski, R.; Steinberg, S. Revisiting the Zintl-Klemm Concept for $\mathrm{ALn}_{2} \mathrm{Ag}_{3} \mathrm{Te}_{5}$-Type Alkaline-Metal (A) Lanthanide (Ln) Silver Tellurides. Crystals 2020, 10, 184. [CrossRef]

25. Göbgen, K.C.; Gladisch, F.C.; Steinberg, S. The Mineral Stutzite: A Zintl-Phase or Polar Intermetallic? A Case Study Using Experimental and Quantum-Chemical Techniques. Inorg. Chem. 2018, 57, 412-421. [CrossRef] [PubMed]

26. Smid, S.; Steinberg, S. Probing the Validity of the Zintl Klemm Concept for Alkaline-Metal Copper Tellurides by Means of Quantum-Chemical Techniques. Materials 2020, 13, 2178. [CrossRef] [PubMed]

27. Mayasree, O.; Sankar, C.R.; Kleinke, K.M.; Kleinke, H. Cu clusters and chalcogen-chalcogen bonds in various copper polychalcogenides. Coord. Chem. Rev. 2012, 256, 1377-1383. [CrossRef]

28. Seong, S.; Albright, T.A.; Zhang, X.; Kanatzidis, M. Te-Te Bonding in Copper Tellurides. J. Am. Chem. Soc. 1994, 116, 7287-7293. [CrossRef]

29. Pyykkö, P. Strong Closed-Shell Interactions in Inorganic Chemistry. Chem. Rev. 1997, 97, 597-636. [CrossRef]

30. Mitchell, K.; Ibers, J.A. Rare-Earth Transition-Metal Chalcogenides. Chem. Rev. 2002, 102, 1929-1952. [CrossRef]

31. WinXPow; Version 2.23; STOE \& Cie GmbH: Darmstadt, Germany, 2008.

32. Match! Version 3.8.0.137; Crystal Impact GbR: Bonn, Germany, 2019.

33. Wang, F.F.Y.; Cox, D.E. The coherent neutron scattering amplitude of Rb: A neutron diffraction study of RbCl. Acta Crystallogr. Sect. A Found. Crystallogr. 1970, 26, 377-379. [CrossRef]

34. Rabadanov, M.K. Anharmonic and "disorder" models of ZnTe structure: High-temperature single-crystal X-ray diffraction studies. Kristallografiya 1995, 40, 505-510.

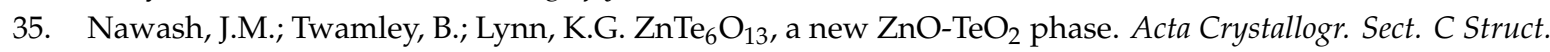
Chem. 2007, 63, i66-i68. [CrossRef] [PubMed]

36. Bruker, A.P.E.X. SAINT+ and SADABS; Bruker AXS Inc.: Madison, WI, USA, 2009.

37. XPREP; Version 6.03; Bruker AXS Inc.: Madison, WI, USA, 2014.

38. Sheldrick, G.M. A short history of SHELX. Acta Crystallogr. Sect. A Found. Crystallogr. 2008, 64, 112-122. [CrossRef] [PubMed]

39. Sheldrick, G.M. Crystal structure refinement with SHELXL. Acta Crystallogr. Sect. C Struct. Chem. 2015, 71, 3-8. [CrossRef]

40. Blöchl, P.E. Projector augmented-wave method. Phys. Rev. B Condens. Matter Mater. Phys. 1994, 50, 17953-17979. [CrossRef] [PubMed]

41. Kresse, G.; Hafner, J. Ab initio molecular dynamics for liquid metals. Phys. Rev. B: Condens. Matter Mater. Phys. 1993, 47, 558-561. [CrossRef]

42. Kresse, G.; Furthmüller, J. Efficiency of ab-initio total energy calculations for metals and semiconductors using a plane-wave basis set. Comput. Mater. Sci. 1996, 6, 15-50. [CrossRef]

43. Kresse, G.; Furthmüller, J. Efficient iterative schemes for ab initio total energy calculations using a plane-wave basis set. Phys. Rev. B Condens. Matter Mater. Phys. 1996, 54, 11169-11186. [CrossRef] [PubMed]

44. Kresse, G.; Joubert, D. From ultrasoft pseudopotentials to the projector augmented-wave method. Phys. Rev. B Condens. Matter Mater. Phys. 1999, 59, 1758-1775. [CrossRef]

45. Kresse, G.; Marsman, M.; Furthmüller, J. Vienna Ab-initio Simulation Package VASP: The Guide; Faculty of Physics, Universität Wien: Vienna, Austria, 2014. 
46. Perdew, J.P.; Burke, K.; Ernzerhof, M. Generalized Gradient Approximation Made Simple. Phys. Rev. Lett. 1996, 77, 3865-3868. [CrossRef] [PubMed]

47. Provino, A.; Steinberg, S.; Smetana, V.; Kulkarni, R.; Dhar, S.K.; Manfrinetti, P.; Mudring, A.-V. Gold-rich $\mathrm{R}_{3} \mathrm{Au}_{7} \mathrm{Sn}_{3}$ : Establishing the interdependence between electronic features and physical properties. J. Mater. Chem. C 2015, 3, 8311-8321. [CrossRef]

48. Smetana, V.; Steinberg, S.; Mudryk, Y.; Pecharsky, V.; Miller, G.J.; Mudring, A.-V. Cation-Poor Complex Metallic Alloys in $\mathrm{Ba}(\mathrm{Eu})-\mathrm{Au}-\mathrm{Al}(\mathrm{Ga})$ Systems: Identifying the Keys that Control Structural Arrangements and Atom Distributions at the Atomic Level. Inorg. Chem. 2015, 54, 10296-10308. [CrossRef] [PubMed]

49. Bigun, I.; Steinberg, S.; Smetana, V.; Mudryk, Y.; Kalychak, Y.; Havela, L.; Pecharsky, V.; Mudring, A.-V. Magnetocaloric Behavior in Ternary Europium Indides EuT 5 In: Probing the Design Capability of First-Principles-Based Methods on the Multifaceted Magnetic Materials. Chem. Mater. 2017, 29, 2599-2614. [CrossRef]

50. Deringer, V.L.; Tchougréeff, A.L.; Dronskowski, R. Crystal Orbital Hamilton Population (COHP) Analysis As Projected from Plane-Wave Basis Sets. J. Phys. Chem. A 2011, 115, 5461-5466. [CrossRef]

51. Dronskowski, R.; Blöchl, P.E. Crystal Orbital Hamilton Populations (COHP). Energy-Resolved Visualization of Chemical Bonding in Solids Based on Density-Functional Calculations. J. Phys. Chem. 1993, 97, 8617-8624. [CrossRef]

52. Steinberg, S.; Dronskowski, R. The Crystal Orbital Hamilton Population (COHP) Method as a Tool to Visualize and Analyze Chemical Bonding in Intermetallic Compounds. Crystals 2018, 8, 225. [CrossRef]

53. Maintz, S.; Deringer, V.L.; Tchougréff, A.L.; Dronskowski, R. Analytic Projection from Plane-Wave and PAW Wavefunctions and Application to Chemcial-Bonding Analysis in Solids. J. Comput. Chem. 2013, 34, 2557-2567. [CrossRef]

54. Maintz, S.; Deringer, V.L.; Tchougréff, A.L.; Dronskowski, R. LOBSTER: A tool to extract chemical bonding from plane-wave based DFT. J. Comput. Chem. 2016, 37, 1030-1035. [CrossRef]

55. Ertural, C.; Steinberg, S.; Dronskowski, R. Development of a robust tool to extract Mulliken and Löwdin charges from plane waves and its application to solid-state materials. RSC Adv. 2019, 9, 29821-29830. [CrossRef]

56. Eck, B. wxDragon 2.2.3; RWTH Aachen University: Aachen, Deutschland, 2020.

57. Mansuetto, M.F.; Keane, P.M.; Ibers, J.A. Synthesis, Structure, and Conductivity of the New Group IV Chalcogenides, $\mathrm{KCuZrQ}_{3}(Q=\mathrm{S}$, Se, Te). J. Solid State Chem. 1992, 101, 257-264. [CrossRef]

58. Koscielski, L.A.; Ibers, J.A. The Structural Chemistry of Quaternary Chalcogenides of the Type $A_{M M} Q_{3}$. Z. Anorg. Allg. Chem. 2012, 638, 2585-2593. [CrossRef]

59. Yao, J.; Deng, B.; Sherry, L.J.; McFarland, A.D.; Ellis, D.E.; Van Duyne, R.P.; Ibers, J.A. Syntheses, Structure, Some Band Gaps, and Electronic Structures of CsLnZnTe 3 (Ln = La, Pr, Nd, Sm, Gd, Tb, Dy, Ho, Er, Tm, Y). Inorg. Chem. 2004, 43, 7735-7740. [CrossRef]

60. Cordero, B.; Gómez, V.; Platero-Prats, A.E.; Revés, M.; Echeverría, J.; Cremades, E.; Barragán, F.; Alvarez, S. Covalent radii revisited. Dalton Trans. 2008, 2008, 2832-2838. [CrossRef] [PubMed]

61. Rustige, C.; Brühmann, M.; Steinberg, S.; Meyer, E.; Daub, K.; Zimmermann, S.; Wolberg, M.; Mudring, A.-V.; Meyer, G. The Prolific $\left\{Z R_{6}\right\} X_{12} R$ and $\left\{Z R_{6}\right\} X_{10}$ Structure Types with Isolated Endohedrally Stabilized $(Z)$ Rare-Earth Metal (R) Cluster Halide (X) Complexes. Z. Anorg. Allg. Chem. 2012, 638, 1922-1931. [CrossRef]

62. Provino, A.; Steinberg, S.; Smetana, V.; Paramanik, U.; Manfrinetti, P.; Dhar, S.K.; Mudring, A.-V. Gold in the Layered Structures of $\mathrm{R}_{3} \mathrm{Au}_{7} \mathrm{Sn}_{3}$ : From Relativity to Versatility. Cryst. Growth Des. 2016, 16, 5657-5668. [CrossRef]

63. Tappe, F.; Pöttgen, R. Rare earth-transition metal-cadmium intermetallics-Crystal chemistry and physical properties. Rev. Inorg. Chem. 2011, 31, 5-25. [CrossRef]

64. Steinberg, S.; Brgoch, J.; Miller, G.J.; Meyer, G. Identifying a Structural Preference in Reduced Rare-Earth Metal Halides by Combining Experimental and Computational Techniques. Inorg. Chem. 2012, 51, 11356-11364. [CrossRef] [PubMed]

65. Bell, T.; Steinberg, S.; Meyer, G. Electron Counting Rules and Electronic Structure in Tetrameric Transition-Metal (T)-Centered Rare-Earth (R) Cluster Complex Halides (X). Inorg. Chem. 2015, 54, 1026-1037. [CrossRef] 
66. Lin, X.; Tabassum, D.; Mar, A. Narrowing the gap: From semiconductor to semimetal in the homologous series of rare-earth zinc arsenides $\mathrm{RE}_{2-y} \mathrm{Zn}_{4} \mathrm{As}_{4} \cdot n$ (REAs) and Mn-substituted derivatives $\mathrm{RE}_{2-y} \mathrm{Mn}_{x} \mathrm{Zn}_{4-x} \mathrm{As}_{4} \cdot n$ (REAs) (RE = La-Nd, Sm, Gd). Dalton Trans. 2015, 44, 20254-20264. [CrossRef]

67. Kim, S.-J.; Kraus, F.; Fässler, T.F. $\mathrm{Na}_{6} \mathrm{ZnSn}_{2}, \mathrm{Na}_{4.24} \mathrm{~K}_{1.76(1)} \mathrm{ZnSn}_{2}$, and $\mathrm{Na}_{20} \mathrm{Zn}_{8} \mathrm{Sn}_{11}$ : Three Intermetallic Structures Containing the Linear $\{\mathrm{Sn}-\mathrm{Zn}-\mathrm{Sn}\}^{6-}{ }^{6}$ Unit. J. Am. Chem. Soc. 2009, 131, 1469-1478. [CrossRef]

68. Simon, A.; Mattausch, H.; Ryazanov, M.; Kremer, R.K. Lanthanides as d Metals. Z. Anorg. Allg. Chem. 2006, 632, 919-929. [CrossRef]

69. Corbett, J.D. Exploratory Synthesis: The Fascinating and Diverse Chemistry of Polar Intermetallic Phases. Inorg. Chem. 2010, 49, 13-28. [CrossRef]

70. Gladisch, F.C.; Steinberg, S. Revealing Tendencies in the Electronic Structures of Polar Intermetallic Compounds. Crystals 2018, 8, 80. [CrossRef]

71. Lin, Q.; Miller, G.J. Electron-Poor Polar Intermetallics: Complex Structures, Novel Clusters, and Intriguing Bonding with Pronounced Electron Delocalization. Acc. Chem. Res. 2018, 51, 49-58. [CrossRef] [PubMed]

(C) 2020 by the authors. Licensee MDPI, Basel, Switzerland. This article is an open access article distributed under the terms and conditions of the Creative Commons Attribution (CC BY) license (http://creativecommons.org/licenses/by/4.0/). 